\title{
Suitability versus fidelity for rating single-photon guns
}

\author{
George M. Hockney, Pieter Kok, and Jonathan P. Dowling \\ Quantum Computing Technologies Group, Section 367, Jet Propulsion Laboratory, California Institute of Technology \\ Mail Stop 126-347, 4800 Oak Grove Drive, Pasadena, California 91109
}

(Version: October 30, 2018)

\begin{abstract}
The creation of specified quantum states is important for most, if not all, applications in quantum computation and communication. The quality of the state preparation is therefore an essential ingredient in any assessment of a quantum-state gun. We show that the fidelity, under the standard definitions is not sufficient to assess quantum sources, and we propose a new measure of suitability that necessarily depends on the application for the source. We consider the performance of single-photon guns in the context of quantum key distribution (QKD) and linear optical quantum computation. Single-photon sources for QKD need radically different properties than sources for quantum computing. Furthermore, the suitability for single-photon guns is discussed explicitly in terms of experimentally accessible criteria.
\end{abstract}

PACS numbers: 03.67.-a, 03.65.Wj, 03.67.Dd, 03.67.Hk

One of the requirements for quantum computation and communication is the ability to faithfully produce certain input states [1]. For quantum computers in general, this means that we have to be able to initialize the registers in some $|0\rangle$ state. Quantum communication involves the transmission of quantum states, and the quality of the state preparation determines in part the success of the communication.

Optical implementations of quantum communication and computation such as cryptography, teleportation, linear optical quantum computers, interferometric quantum non-demolition measurements, and many other applications, often rely on good single-photon sources [2]. There are many proposals for single-photon guns, ranging from semiconductor quantum dots and manipulated individual molecules to parametric down-converters [3], but the suitability of these sources has not yet been sufficiently addressed. Whereas attenuated coherent states might be a good approximation to a single-photon state for some applications, its two-photon contribution renders it unsuitable for cryptography [4]. Any measure of suitability therefore has to take the intended purpose of the state into account.

One possible choice for the suitability of a source would be the fidelity $f_{A B}$ of the input state, with density matrix $\rho_{A}$, with respect to the desired state $\rho_{B}$ : $f_{A B} \equiv\left\{\operatorname{Tr}\left[\left(\sqrt{\rho_{A}} \rho_{B} \sqrt{\rho_{A}}\right)^{1 / 2}\right]\right\}^{2}[5]$. This fidelity satisfies $0 \leq f_{A B} \leq 1$, and most importantly, $f_{A B}=1$ if and only if $\rho_{A}=\rho_{B}$. When one of the systems is in a pure state $|\psi\rangle$ and the other system is in a mixed state $\rho$, this measure reduces to $\operatorname{Tr}(\rho|\psi\rangle\langle\psi|)$. However, $f_{A B}$ is generally not a good measure for the suitability of a state-preparation device (such as a single-photon gun) given a specific application. The reason is that several different input states may be equally suitable for a specific application. When two distinct states $\rho_{X}$ and $\rho_{Y}$ are both useful states for a specific application, a state $\rho_{A}$ might have a large $f_{X A}$, but a small $f_{Y A}$. Similarly, a state $\rho_{B}$ might have a small $f_{X B}$, but a large $f_{Y B}$. Both $\rho_{A}$ and $\rho_{B}$ are suitable for the application, but the fidelity acknowledges only one of them.

In this paper we propose a general definition for the suitability $S_{G T}$ of a source gun $G$ given a target application $T$. The suitability satisfies $0 \leq S_{G T} \leq 1$, and is a good measure of how well a candidate gun will work in a given target application. We will first give an example where the fidelity breaks down as a performance measure of the single-photon gun, and subsequently we define the general suitability $S_{G T}$. We then use this formalism to describe single-photon guns in quantum key distribution (QKD) and demonstrate how a source highly suitable for QKD is not at all suitable for quantum teleportation.

To understand why conventional definitions of the fidelity are problematic, consider the QKD protocol associated with the 1984 cryptographic scheme by Bennett and Brassard [6]. In this application, Alice chooses a random string from a set of four pair-wise orthogonal polarization states $\mathcal{S}=\{|H\rangle,|V\rangle,|L\rangle,|R\rangle\}$. She then prepares and sends these states to Bob. In order to ensure security, it is important that the states only contain a single photon [4]. However, it is not important that the state's frequency or exact timing within a window is controlled (as long as this is not correlated with the polarization). Pure states with some spread in time and frequency are as suitable for key distribution as a mixed state, provided the polarization is definite and the state contains exactly one photon.

Suppose for simplicity that the unknown and unimportant part of the state can be in only three (pure) states $|A\rangle,|B\rangle$, or $|C\rangle$. When Alice wishes to send the state $|\psi\rangle \in \mathcal{S}$ to Bob, it does not matter whether the state she produces is actually $|\psi\rangle \otimes|A\rangle,|\psi\rangle \otimes|B\rangle$, $|\psi\rangle \otimes|C\rangle$ or a combination of these. Although one could require Alice to create the state $|\psi\rangle \otimes|A\rangle$, this is overly restrictive. In a real system this would be equivalent to requiring the input gun to have no time 
jitter or any other irrelevant mixing. It only matters that $|\psi\rangle$ is the correct polarization and has only one photon. When we define Alice's target state as $\rho_{T}=$ $\frac{1}{3}|\psi, A\rangle\left\langle\psi, A\left|+\frac{1}{3}\right| \psi, B\right\rangle\left\langle\psi, B\left|+\frac{1}{3}\right| \psi, C\right\rangle\langle\psi, C|, \operatorname{Tr}\left(\rho_{G} \rho_{T}\right)$ is always smaller than $1 / 3$ and yields different values for equally useful input states. Using the fidelity instead, namely, $f_{A B}=\left\{\operatorname{Tr}\left[\left(\sqrt{\rho_{A}} \rho_{B} \sqrt{\rho_{A}}\right)^{1 / 2}\right]\right\}^{2}$, also fails, since fidelity is only a measure of how much two states are alike. $f_{A B}$ is unity only if the input state is exactly $\rho_{T}$, but any of several other states are equally suitable.

The solution is to define a procedure for writing a target state $\rho_{T}$, a gun state $\rho_{G}$, and an expression $S\left(\rho_{T}, \rho_{G}\right)$ that has the desired propery of being close to one if and only if the gun is suitable for the target. We define $\rho_{G}$, the gun state, simply as the state that produced by the gun. The target state $\rho_{T}$ is a bit subtler because it is not a physical state in the system. The procedure for defining $\rho_{T}$ is to identify all states which would be useable gun states, find a set of states spanning that space, and then define $\rho_{T}$ as a complete mixture of those states. That is, given a complete set of $N$ suitable states $\left|\phi_{i}\right\rangle$, the target density matrix would be $\rho_{T} \equiv N^{-1} \sum_{i}^{N}\left|\phi_{i}\right\rangle\left\langle\phi_{i}\right|$. In the QKD example, this leads to Alice's target state $\rho_{T}=\frac{1}{3}|\psi, A\rangle\left\langle\psi, A\left|+\frac{1}{3}\right| \psi, B\right\rangle\left\langle\psi, B\left|+\frac{1}{3}\right| \psi, C\right\rangle\langle\psi, C|$. To define the suitability of a quantum source, let $F_{A B} \equiv$ $\operatorname{Tr}\left(\rho_{A} \rho_{B}\right)$ for any two normalized density matrices $\rho_{A}$ and $\rho_{B}$. Given two mixtures we have $F_{A B} \leq F_{A A}$. Note that $F$ is not a proper definition of fidelity, since $F_{A A} \neq 1$ if $\rho_{A}$ is not a pure state.

We propose the following definition for the suitability $S$ of a gun that purports to create a particular quantum state:

$$
S_{G T} \equiv \frac{F_{G T}}{F_{T T}},
$$

where $\rho_{G}$ is the output state of the gun and $\rho_{T}$ is a mixture of all possible target states associated with a particular application. If there is one and only one pure target state, then $F_{T T}=1$ and $S_{G T}=f_{G T}$. For any input state that completely overlaps the requirement, this definition of $S$ yields unity. This may be explicitly worked out for the three states mentioned in the QKD example above, yielding $F_{T T}=\frac{1}{3}$ and $S_{G T}=1$, as advertised.

Since $\rho_{T}$ is a complete mixture on a subspace, the triangle identity yields

$$
S_{G T}=\frac{F_{G T}}{F_{T T}} \leq \frac{F_{G G}}{F_{T T}} .
$$

Since $F_{G G}$ is determined only by the gun, this leads to an important measure of the quality of the gun that only depends on the output state of the gun. If there is only one suitable target state (i.e., $\rho_{T}$ is pure), then we have $F_{T T}=1$ and $S_{G T}$ reduces to $F_{G T}$. This must be less than $F_{G G}$, and $F_{G G}$ therefore directly limits the suitability of a candidate gun for this class of targets.
So far, the formalism has been completely general, and we will now consider the important special case of singlephoton guns. Naively, an ideal single-photon gun is a device that emits one and only one photon with a particular frequency in a given spatial mode when triggered to do so. Such a device can not exist. According to Heisenberg's uncertainty relation in energy and time, it is not possible to fix both the frequency and the triggering time with infinite precision. This means that we have to admit a continuum of frequency modes if we keep the photon number fixed in a given time window. The difficulties involving ideal single-photon guns are closely related to the fact that single photons do not have a well-defined wave function [7]. We therefore have to be careful when we define the single-photon states that are produced by the guns. In particular, the desired states differ from application to application.

For single-photon guns, we can modify $F_{A B}$ such that we obtain $F_{A B}^{(1)} \equiv \operatorname{Tr}\left(\rho_{A}|1\rangle\langle 1| \rho_{B}\right)$, where $|1\rangle\langle 1| \equiv$ $\int d \vec{k} d \omega \hat{a}_{\vec{k}}^{\dagger}(\omega)|0\rangle\langle 0| \hat{a}_{\vec{k}}(\omega)$. Here, $\hat{a}_{\vec{k}}^{\dagger}(\omega)$ and $\hat{a}_{\vec{k}}(\omega)$ are the creation and annihilation operators satisfying $\left[\hat{a}_{\vec{k}}(\omega), \hat{a}_{\vec{k}^{\prime}}^{\dagger}\left(\omega^{\prime}\right)\right]=\delta\left(\vec{k}-\vec{k}^{\prime}\right) \delta\left(\omega-\omega^{\prime}\right)$. Obviously, we have $F_{A B}^{(1)} \leq F_{A B}$. Inserting $|1\rangle\langle 1|$ does not affect $F_{G T}$ or $F_{T T}$, since $\rho_{T}$ is already confined to the $|1\rangle\langle 1|$ subspace. However, $F_{G G}^{(1)}$ gives a better measure for the performance of single-photon guns than $F_{G G}$, since $F_{G G}^{(1)} \leq F_{G G}$, and therefore $S_{G T} \leq F_{G G}^{(1)} / F_{T T}$.

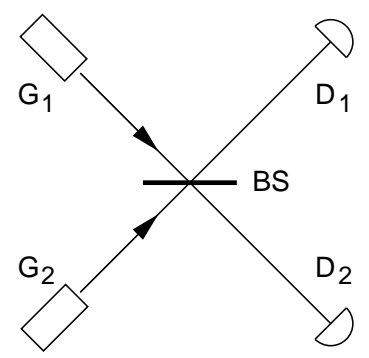

FIG. 1. The Hong-Ou-Mandel test: $G_{1}$ and $G_{2}$ are the single-photon guns and $D_{1}$ and $D_{2}$ are the photodetectors. When the output modes of the guns are mixed in the beam splitter (BS), there should be no detector coincidences in $D_{1}$ and $D_{2}$.

A good approximation of $F_{G G}^{(1)}$ can be measured using a Hong-Ou-Mandel (HOM) interferometer (see Fig. 1). In this measurement, the input of two identical singlephoton guns $G_{1}$ and $G_{2}$ is directed toward a beam splitter and then detected by two ideal photodetectors $D_{1}$ and $D_{2}$. Assuming the input state from each gun was a single photon in the same state, the output state after the beam splitter is $|2,0\rangle-|0,2\rangle$. However, if the input state is a mixed state or if the two guns are not identical, there will be contamination of $|1,1\rangle$ states in the output.

The ability to trigger the gun reproducibly is an impor- 
tant component of this test. If it is not possible to obtain a high $F_{G G}^{(1)}$, then the suitability of the candidate gun for any application requiring a pure state will be low. If the application only requires a mixed state (in, for example, the case of quantum key distribution above), then the gun may still be suitable. This is a clear example where two different applications yield different suitabilities for the same single-photon gun.

It is instructive to apply the suitability formalism to the HOM experiment itself, because that demonstrates how to apply the concept of suitability when there are two inputs. Let the input state on the beam splitter be $\rho_{\text {in }}$ and the output state $\rho_{\text {out }}$. The objective of the experiment is to obtain a high visibility in the interference, i.e., to maximize $v=1-\left\langle 1,1\left|\rho_{\text {out }}\right| 1,1\right\rangle$, where $|1,1\rangle$ denotes the state that yields a detector coincidence in $D_{1}$ and $D_{2}$.

We now want to find the target state $\rho_{T}$ that is to be used in determining the suitability of the single-photon guns for the HOM experiment. On the detector side of the beam splitter, a suitable state is either two photons in one detector or two photons in the other. If we label the two detectors $a$ and $b$ this is simply

$$
\begin{aligned}
\rho_{T}=\int\{ & {\left[\hat{a}_{\vec{k}}^{\dagger}(\omega) \hat{a}_{\overrightarrow{k^{\prime}}}^{\dagger}\left(\omega^{\prime}\right)|0\rangle\langle 0| \hat{a}_{\vec{k}}(\omega) \hat{a}_{\overrightarrow{k^{\prime}}}\left(\omega^{\prime}\right)\right]+} \\
& {\left.\left[\hat{b}_{\vec{k}}^{\dagger}(\omega) \hat{b}_{\overrightarrow{k^{\prime}}}^{\dagger}\left(\omega^{\prime}\right)|0\rangle\langle 0| \hat{b}_{\vec{k}}^{\dagger}(\omega) \hat{b}_{\overrightarrow{k^{\prime}}}^{\dagger}\left(\omega^{\prime}\right)\right]\right\} d X, }
\end{aligned}
$$

where $\hat{a}_{\vec{k}}$ and $\hat{b}_{\vec{k}}$ are the annihilation operators associated with the detectors, and $d X \equiv$ $h(\vec{k}, \omega) h\left(\overrightarrow{k^{\prime}}, \omega^{\prime}\right) d \vec{k} d \omega d \overrightarrow{k^{\prime}} d \omega^{\prime}$. Here $h(\vec{k}, \omega)$ limits the range of $k$ and $\omega$ accepted by the beam splitter. This is the target on the detector side of the beam splitter. We would prefer to have the target on the source side of the beam splitter, and because the beam splitter is presumed to be lossless this won't change any suitability (because of the asymmetry between the source and target, any losses must be computed as part of the source). If the inputs are $c$ and $d$ this becomes

$$
\begin{aligned}
\rho_{T}=\int & \left\{\left[\hat{c}_{\vec{k}}^{\dagger}(\omega) \hat{d}_{\overrightarrow{k^{\prime}}}^{\dagger}\left(\omega^{\prime}\right)|0\rangle\langle 0| \hat{c}_{\vec{k}}(\omega) \hat{d}_{\overrightarrow{k^{\prime}}}\left(\omega^{\prime}\right)\right]+\right. \\
& \left.\frac{1}{2}\left[\hat{c}_{\vec{k}}^{\dagger}(\omega) \hat{d}_{\overrightarrow{k^{\prime}}}^{\dagger}\left(\omega^{\prime}\right)+\hat{d}_{\vec{k}}^{\dagger}(\omega) \hat{c}_{\overrightarrow{k^{\prime}}}^{\dagger}\left(\omega^{\prime}\right)\right)\right]|0\rangle \\
& \left.\times\langle 0|\left[\left(\hat{c}_{\vec{k}}(\omega) \hat{d}_{\overrightarrow{k^{\prime}}}\left(\omega^{\prime}\right)+\hat{d}_{\vec{k}}(\omega) \hat{c}_{\overrightarrow{k^{\prime}}}\left(\omega^{\prime}\right)\right)\right]\right\} d X
\end{aligned}
$$

This includes both the expected $|1,1\rangle$ inputs as well as $|2,0\rangle+|0,2\rangle$, an eigenvector of the beam splitter. For the purpose of testing two independent single-photon guns it is easy to believe the entangled state will not be generated, and so the HOM test shows how well a pure $|1,1\rangle$ state was generated.

The input state $\rho_{G}=\rho_{G 1} \otimes \rho_{G 2}$ is the physical state produced when the experimenter pushes the button telling both guns to fire. The suitability $S_{G T}=F_{G T} / F_{T T}$ is now close to unity if have both guns individually generate the same pure state. Other suitable systems may involve either entangled or classical correlations between the two guns, such as in parametric down-conversion. All of the required information is always in the state $\rho_{G}$ of the two-gun output. If the guns are independent it is important that each gun's output be a pure state and not a mixed state traced over entangled partners left in the gun.

As an example, the formalism developed above is used to design a QKD system using spontaneous parametric down-conversion in continuous wave operation (see Fig. 2a). A (nearly perfect) photodetector in the idler mode counts the number of photons per time interval. When the outcome is " 1 ", the same time interval in the signal mode then also contains exactly one photon in a known polarization. Alice will rotate this photon into one of the states $|\psi\rangle \in \mathcal{S}$ and send it to Bob (see Fig. 2b). The state sent to Bob will have a low $F_{G G}^{(1)}$ but high $S_{G T}$. The source will have high immunity to eavesdropping attacks, which means that it has a low suitability $S_{G E}$ for the eavesdropper (where the target state $\rho_{E}$ is constructed for maximum possible information gain by Eve). The state $\rho_{G}$ is now a highly mixed state that contains only one photon (if $D$ is nearly perfect) in the state $|\psi\rangle$, but crossed with a large subspace $\mathcal{R}=\{|A\rangle,|B\rangle,|C\rangle \ldots\}$, representing the unknown time of emission and uncertainty in frequency. This state has a small $F_{G G}^{(1)}$, as may be demonstrated experimentally in a Hong-Ou-Mandel experiment. The target state $\rho_{T}$ required for QKD is the mixed state $|\psi\rangle\langle\psi| \otimes \rho_{\mathcal{R}}$, where $\rho_{\mathcal{R}}=\mathbb{1} / d$ is the identity operator on the subspace $\mathcal{R}$ with dimension $d$. It is clear that $F_{G G}^{(1)} \approx 1 / d$ but so are $F_{T T}$ and $F_{G T}$. Therefore, this gun has $S_{G T} \approx 1$ and is suitable for QKD.

a)

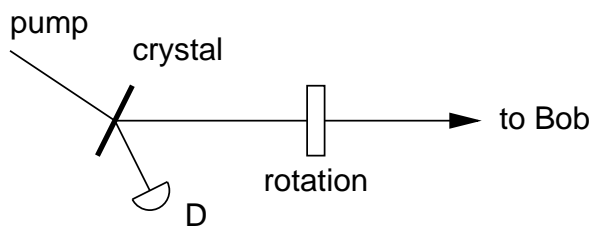

b)

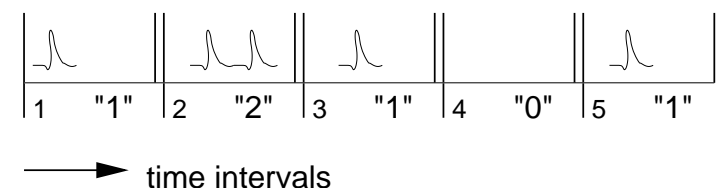

FIG. 2. Spontaneous parametric down-conversion in continuous-wave operation. a) The pump beam created photon pairs in two spatial modes, one of which is continuously monitored. b) The photon counts in the time windows. Only when one photon per time interval is counted by the detector $D$, we deem the creation of a "single-photon state" a success. 
Let us now consider the suitability of the single-photon gun for an eavesdropper Eve. Since Eve does not know the polarization chosen by Alice, she must assign her own density matrix $\sigma_{G}$ (i.e., her knowledge about the protocol) to the system she intercepts. For clarity, $\rho$ is the state according to Alice, whereas $\sigma$ is the state according to Eve. For example, Alice creates a state with definite polarization (say $\rho=|\psi\rangle\langle\psi|$ ), whereas Eve must describe it as a maximally mixed state in the polarization $(\sigma=\mathbb{1} / 2)$. Eve can gain information about the key when $\sigma$ is no longer of this form.

The target density operator $\sigma_{E}$ is then defined such that it allows Eve to obtain maximal information about the string of qubits that Alice sent to Bob. The quantity $S_{G E}=S\left(\sigma_{G}, \sigma_{E}\right)$ is the suitability of $\sigma_{G}$ for eavesdropping. Perfect key distribution is defined as $S_{G E}=0$. Notice that $S_{G E}$ is not simply related to $S_{G T}$. For example, the source may sometimes fail to emit anything, which reduces both $S_{G T}$ and $S_{G E}$. In this case a small $S_{G T}$ does not necessarily mean that eavesdropping is possible. Suppose that in a practical system based on this scheme the main imperfection is the detector inefficiency (and not the possible correlations between $\mathcal{S}$ and $\mathcal{R}$ ). Then it is easy to calculate how a given efficiency of $D$ affects both $S_{G T}$ and $S_{G E}$. Due to an imperfect detector, the state $\rho_{G}$ acquires a contamination of two-photon states at a fraction $\epsilon$, which would make $S_{G T}=1-\epsilon$ and $S_{G E}=\epsilon$. This analysis would be completely different for other applications. Suppose that a pure target state were required for some protocol, for example in quantum teleportation. As may be seen in the Hong-Ou-Mandel experiment, the continuous wave single-photon gun based on down-conversion has an unmeasureably small $F_{G G}^{(1)}$. Therefore, such a gun would be useless for any protocol requiring a pure target state because $F_{T T} \simeq 1$ and $S_{G T} \leq F_{G G}^{(1)}$. The suitability of a single-photon gun therefore depends critically on the application.

In conclusion, we have shown that the performance of a quantum source must be evaluated in the context of a specific application. The suitability of a source cannot be defined as the fidelity of the output state with a target state. The reason is that there might be multiple distinct target states all equally suited for the application. We defined the suitability $S_{G T}=\operatorname{Tr}\left(\rho_{G} \rho_{T}\right) / \operatorname{Tr}\left(\rho_{T} \rho_{T}\right)$ instead, where $\rho_{G}$ is the output state of the gun and $\rho_{T}$ is an equal mixture of all suitable target states. In the case where $\rho_{T}$ is pure the suitability reduces to the standard fidelity.

We explicitly investigated the case where the quantum source is a single-photon gun. It was shown that for QKD, a system using a continuously pumped SPDC crystal can provide the single-photon states needed to prevent eavesdropping even though this system would not be useful for other applications where $F_{T T}$ is close to unity. It is important to carefully understand whether a given application using single-photon quantum optics requires a pure state or can be run with any of a number of states. The requirements on the photon source are very different in these two cases. For many applications, an analysis similar to the one done for the Hong-Ou-Mandel visibility requirement must be done. This is because the apparatus uses several input photons in different places, and $G$ is the combined state of all of them. In these applications it is tempting to require that all the guns emit maximally overlapping pure states. However, this is not necessary if there is a correlation between the inputs.

This work was carried out at the Jet Propulsion Laboratory, California Institute of Technology, under a contract with the National Aeronautics and Space Administration. The authors wish to thank Deborah Jackson for many useful discussions. P.K. acknowledges the United States National Research Council. Support was received from the Office of Naval Research, the Advanced Research and Development Activity, the National Security Agency, and the Defense Advanced Research Projects Agency.

[1] D.P. DiVincenzo, Fortschr. Phys. 48, 771 (2000); also in: Scalable Quantum Computers, S.L. Braunstein and H.-K. Lo (Eds.), pp. 1-13, Wiley-VCH (2001).

[2] D. Bouwmeester et al., Nature 390, 575 (1997); E. Knill et al., Nature 409, 46 (2001); P. Kok et al., quant$\mathrm{ph} / 0202046$.

[3] A. Beveratos et al., Eur. Phys. J. D 18, 191 (2002); Z.L. Yuan et al., Science 295, 102 (2002); E. Moreau et al., App. Phys. Lett. 79, 2865 (2001); K.O. Greulich and E. Thiel, Single Mol. 2, 5 (2001); P. Michler et al., Science 290, 2282 (2000); M. Lau, Mrs. Bull. 25, 5 (2000); B. Lounis et al., J. Phys. IV 10, 13 (2000); C.L. Foden et al., Phys. Rev. A 62, 011803 (2000); C. Brunel et al., Cr. Acad. Sci. II B 326, 911 (1998); K.M. Gheri et al., Phys. Rev. A 58, R2627 (1998).

[4] G. Brassard, N. Lutkenhaus, T. Mor, and B.C. Sanders, Phys. Rev. Lett. 85, 1330 (2000).

[5] Jozsa, R. J. Mod. Optics 41, 2315-2323 (1994).

[6] C.H. Bennett and G. Brassard, Proc. IEEE Int. Conf. Comp., IEEE New York (1984).

[7] P.A.M. Dirac, Proc. R. Soc. London A 114, 243 (1928); K.W. Chan, C.K. Law, and J.H. Eberly, Phys. Rev. Lett. 88, 100402 (2002). 